\title{
LINDENBAUM ALGEBRAS AND PARTIAL CONSERVATIVITY
}

\author{
CHRISTIAN BENNET ${ }^{1}$
}

\begin{abstract}
A partial Lindenbaum algebra $\Gamma^{A}$, where $A$ is a theory extending Peano arithmetic and $\Gamma \in\left\{\Pi_{n}, \Sigma_{n}\right\}$, is the full Lindenbaum algebra for $A$ restricted to sentences in $A$ provably equivalent to $\Gamma_{n}$-sentences. Using a new result on pairs of partially conservative sentences, we show that $\Pi_{n}^{A}$ and $\Sigma_{n}^{A}$ are not isomorphic.
\end{abstract}

1. Introduction. In the following $A$ and $B$ will be any consistent r.e. extensions of $P$ (Peano arithmetic). Let $\sim_{A}$ be the equivalence relation defined by

$$
\varphi \sim_{A} \psi \text { iff } A \vdash \varphi \leftrightarrow \psi .
$$

A partial Lindenbaum algebra $E\left(\Gamma_{n}\right)$ for $A$ is the lattice of associated equivalence classes $[\varphi]$, where $\Gamma \in\{\Sigma, \Pi, \Delta\}$ and $\varphi \in \Gamma_{n}$ (here and in what follows we assume that $n \geq 1$ ), with $\cup$ and $\cap$ defined by

$$
[\varphi] \cup[\psi]=[\varphi \vee \psi], \quad[\varphi] \cap[\psi]=[\varphi \wedge \psi] .
$$

It is well known that the full Lindenbaum algebras for extensions of $P$ are all isomorphic. The main result of $\S 3$ is that this is not the case for partial Lindenbaum algebras. In $\S 2$ we show some results on partially conservative sentences which are used later on. Notions not defined in the paper are standard and may be found in [2 or 4]. We will freely use Craig's theorem (cf. [2]) and constructions via diagonalization.

2. Pairs of partially conservative sentences. A sentence $\varphi$ is $\Gamma_{n}$-conservative over $A, \varphi \in \operatorname{Cons}\left(\Gamma_{n}, A\right)$, if for all $\psi \in \Gamma_{n}$,

$$
\text { if } A+\varphi \vdash \psi \text { then } A \vdash \psi \text {. }
$$

This notion was introduced by Guaspari in [3].

THEOREM 1. There are sentences $\sigma_{0}, \sigma_{1} \in \Sigma_{n}$ such that

(i) $A \nvdash \sigma_{0} \vee \sigma_{1}$,

(ii) $\sigma_{i} \in \operatorname{Cons}\left(\Pi_{n}, A+\neg \sigma_{1-i}\right)$ for $i=0,1$.

In order to prove this we need some notation and two lemmas. Let $\left[\Gamma_{n}\right](x, y, z)$ be the formula

$$
\Gamma_{n}(y) \wedge \operatorname{Prf}_{\alpha+x}(y, z) \rightarrow \Gamma_{n} \text {-true }(y),
$$

where $\alpha(v)$ is a p.r. binumeration of (the axioms of) $A, \alpha+x$ is the formula $\alpha(v) \vee v=x$, and $\Gamma_{n}$-true $(y)$ is a partial truth definition for $\Gamma_{n}$-sentences, i.e. $p \vdash \varphi \leftrightarrow \Gamma_{n}$-true $(\bar{\varphi})$ for every $\varphi \in \Gamma_{n}$. The following facts concerning the formula $\left[\Gamma_{n}\right](x, y, z)$ are easy to prove.

Received by the editors March 29, 1985.

1980 Mathematics Subject Classification. Primary 03F25, 03 F30.

${ }^{1}$ I would like to thank Per Lindström for useful comments and advice. 
LEMMA 1. (i) $\Gamma_{n}(x, y, z)$ is a $\Gamma_{n}$-formula.

(ii) For all $\varphi, m, A+\varphi \vdash \forall u, v \leq \bar{m}\left[\Gamma_{n}\right](\bar{\varphi}, u, v)$.

(iii) If $\psi$ is a $\Gamma_{n}$-sentence and $p$ is (the Gödel number of) a proof of $\psi$ in $A+\varphi$, then $A+\neg \psi \vdash \neg\left[\Gamma_{n}\right](\bar{\varphi}, \bar{\psi}, \bar{p})$.

We shall also need the following lemma.

LEMma 2. If $X$ is r.e., then there are $\Sigma_{n}$-formulae $\theta_{0}(x), \theta_{1}(x)$ satisfying, for $i=0,1$,

(i) $A+\theta_{i}(\bar{k}) \vdash \neg \theta_{1-i}(\bar{k})$ for all $k$.

(ii) If $k \in X$, then $A \vdash \neg \theta_{i}(\bar{k})$.

(iii) If $k \notin X$, then $\theta_{i}(\bar{k}) \in \operatorname{Cons}\left(\Pi_{n}, A+\neg \theta_{1-i}(\bar{k})\right)$.

Proof. Let $G(x, y)$ be a p.r. relation such that $X=\{k: \exists m G(k, m)\}$, and let $\gamma(x, y)$ be a p.r. binumeration of $G$. For $i=0,1$ let $\xi_{i}(x), \varsigma_{i}(x, y)$, and $\theta_{i}(x)$ be, respectively, $\Sigma_{n^{-}}, \Pi_{n-1^{-}}$, and $\Sigma_{n}$-formulae such that the following is provable in $A$ for all $k$ :

$$
\begin{aligned}
& \left.\xi_{i}(\bar{k}) \leftrightarrow \exists z(\exists u, v \leq z\urcorner\left[\Pi_{n}\right]\left(\overline{\theta_{i}(\bar{k})}, u, v\right) \wedge \forall u \leq z \neg \gamma(\bar{k}, u)\right), \\
& \xi_{i}(\bar{k}) \leftrightarrow \exists z \zeta_{i}(\bar{k}, z), \\
& \theta_{0}(\bar{k}) \leftrightarrow \exists z\left(\varsigma_{0}(\bar{k}, z) \wedge \forall y \leq z \neg \zeta_{1}(\bar{k}, y)\right), \\
& \theta_{1}(\bar{k}) \leftrightarrow \exists z\left(\varsigma_{1}(\bar{k}, z) \wedge \forall y<z \neg \zeta_{0}(\bar{k}, y)\right) .
\end{aligned}
$$

A standard argument now shows that (i) holds.

To prove (ii), suppose $k \in X$. Then there is an $m$ such that $A \vdash \gamma(\bar{k}, \bar{m})$. But since, by Lemma 1 (ii),

$$
A+\theta_{i}(\bar{k}) \vdash \forall u, v \leq \bar{m}\left[\Pi_{n}\right]\left(\overline{\theta_{i}(\bar{k})}, u, v\right),
$$

we have

$$
A+\theta_{i}(\bar{k}) \vdash \neg \xi_{i}(\bar{k})
$$

and thus

$$
A+\theta_{i}(\bar{k}) \vdash \neg \theta_{i}(\bar{k})
$$

from which (ii) follows.

In order to prove (iii), suppose $k \notin X$ and for some $\psi \in \pi_{n}, A+\neg \theta_{1-i}(\bar{k})+\theta_{i}(\bar{k}) \vdash$ $\psi$. Then, by (i), $A+\theta_{i}(\bar{k}) \vdash \psi$. Using Lemma 1(iii) and the fact that $A \vdash \neg \gamma(\bar{k}, \bar{m})$ for all $m$, we have

$$
A+\neg \psi \vdash \xi_{i}(\bar{k})
$$

and therefore

$$
A+\neg \psi \vdash \exists \varsigma_{\varsigma_{i}}(\bar{k}, z)
$$

It is now easy to verify that

$$
A+\neg \psi+\neg \theta_{1-i}(\bar{k}) \vdash \theta_{i}(\bar{k})
$$

and thus

$$
A+\neg \theta_{1-i}(\bar{k}) \vdash \psi
$$

This proves (iii). 
We are now ready for the

Proof of ThEOREM 1. Apply Lemma 2 to $X=\operatorname{Th}(A)$, and let $\varphi$ be such that

$$
A \vdash \varphi \leftrightarrow \theta_{0}(\bar{\varphi}) \vee \theta_{1}(\bar{\varphi})
$$

Since $A$ is consistent we have by Lemma 2 (ii) that $\varphi \notin X$, so

$$
A \nvdash \theta_{0}(\bar{\varphi}) \vee \theta_{1}(\bar{\varphi})
$$

and, by Lemma 2(iii), $\theta_{i}(\bar{\varphi}) \in \operatorname{Cons}\left(\Pi_{n}, A+\neg \theta_{1-i}(\bar{\varphi})\right)$.

In, e.g., [4] several sets of partially conservative sentences are classified as complete $\Pi_{2}^{0}$-sets. In this direction we have

THEOREM 2. The following is a complete $\Pi_{2}^{0}$-set.

$$
\left\{\left(\sigma_{0}, \sigma_{1}\right): \bigwedge_{i=0,1} \sigma_{i} \in \operatorname{Cons}\left(\Pi_{n}, A+\neg \sigma_{1-i}\right) \cap \Sigma_{n}\right\} .
$$

Proof (CF. PRoOF OF LEMMA 5 IN [4]). Let $Y$ be the set in the theorem. Evidently $Y$ is $\Pi_{2}^{0}$. Now let $X$ be any $\Pi_{2}^{0}$-set. We will construct $\Sigma_{n}$-formulae, $\theta_{0}(x)$ and $\theta_{1}(x)$, such that

$$
k \in X \quad \text { iff } \quad\left(\theta_{0}(\bar{k}), \theta_{1}(\bar{k})\right) \in Y .
$$

Let $R(x, y)$ be r.e. and $S(x, y, z)$ be p.r. such that

$$
X=\{k: \forall m R(k, m)\}=\{k: \forall m \exists n S(k, m, n)\}
$$

and let $\sigma(x, y, z)$ be a p.r. binumeration of $S$. For $i=0,1$ we define a number of formulae such that the following is provable in $A$ for all $k, m$.

$$
\begin{gathered}
\gamma_{0, i}(\bar{k}, \bar{m}) \leftrightarrow \forall z\left(\operatorname{Prf}_{\alpha}\left(\overline{\lambda_{i}(\bar{k}, \bar{m})}, z\right) \rightarrow \exists u<z \sigma(\bar{k}, \bar{m}, u)\right), \\
\gamma_{1, i}(\bar{k}, \bar{m}) \leftrightarrow \exists z\left(\sigma(\bar{k}, \bar{m}, z) \wedge \forall u \leq z \neg \operatorname{Prf}_{\alpha}\left(\overline{\lambda_{i}(\bar{k}, \bar{m})}, u\right)\right), \\
\lambda_{i}(\bar{k}, \bar{m}) \leftrightarrow\left(\neg \theta_{1-i}(\bar{k}) \rightarrow \gamma_{0, i}(\bar{k}, \bar{m})\right), \\
\xi_{i}(\bar{k}) \leftrightarrow \exists z\left(\exists u, v \leq z \neg\left[\Pi_{n}\right]\left(\overline{\theta_{i}(\bar{k})}, u, v\right) \wedge \forall u \leq z \gamma_{1, i}(\bar{k}, u)\right), \\
\xi_{i}(\bar{k}) \leftrightarrow \exists z \zeta_{i}(\bar{k}, z), \\
\theta_{0}(\bar{k}) \leftrightarrow \exists z\left(\zeta_{0}(\bar{k}, z) \wedge \forall u \leq z \neg \zeta_{1}(\bar{k}, u)\right), \\
\theta_{1}(\bar{k}) \leftrightarrow \exists z\left(\zeta_{1}(\bar{k}, z) \wedge \forall u<z \neg \zeta_{0}(\bar{k}, u)\right) .
\end{gathered}
$$

This may be done, using Lemma $1(\mathrm{i})$, so that $\gamma_{0, i}(x, y)$ are $\Pi_{1}$-formulae, $\gamma_{1, i}(x, y)$ are $\Sigma_{1}$-formulae, $\xi_{i}(x)$ and $\theta_{i}(x)$ are $\Sigma_{n}$-formulae, and $\zeta_{i}(x, y)$ are $\Pi_{n-1}$-formulae.

Suppose first that $k \in X$. If $A \vdash \theta_{i}(\bar{k})$, it is trivial, using the fact that by (6) and (7) $A \vdash \theta_{i}(\bar{k}) \rightarrow \neg \theta_{1-i}(\bar{k})$ for all $k$, that $\left(\theta_{0}(\bar{k}), \theta_{1}(\bar{k})\right) \in Y$. On the other hand, if $A \forall \theta_{i}(\bar{k})$ the same result holds by an argument similar to that in the proof of Lemma 2(iii).

Suppose now that $k \notin X$. Then, by (4)-(7) and Lemma 1(ii), for all $m$

$$
A+\theta_{i}(\bar{k}) \vdash \gamma_{1, i}(\bar{k}, \bar{m}) \text {. }
$$


By (1) and (2) we have for all $k, m$

$$
A \vdash \gamma_{1, i}(\bar{k}, \bar{m}) \rightarrow \gamma_{0, i}(\bar{k}, \bar{m}) .
$$

So, since $k \notin X$, for some $m_{0}$ such that not $R\left(k, m_{0}\right)$, we have

$$
A+\theta_{i}(\bar{k}) \vdash \gamma_{0, i}\left(\bar{k}, \bar{m}_{0}\right) \text {. }
$$

Further, it is not difficult to show that if not $R(k, m)$ holds and $A+\neg \theta_{1-i}(\bar{k})$ is consistent, then

$$
A+\neg \theta_{1-i}(\bar{k}) \nvdash \gamma_{0, i}(\vec{k}, \bar{m}) .
$$

But then, in order to prove that $\left(\theta_{0}(\bar{k}), \theta_{1}(\bar{k})\right) \notin Y$, we need only show that $A \nvdash$ $\theta_{1-i}(\bar{k})$. But we have already shown that

$$
A+\theta_{i}(\bar{k}) \vdash \gamma_{0, i}\left(\bar{k}, \bar{m}_{0}\right)
$$

so if $A \vdash \theta_{i}(\bar{k})$, then $A+\neg \theta_{1-i}(\bar{k})$ is inconsistent. Again using the fact that

$$
A \vdash \theta_{i}(\bar{k}) \rightarrow \neg \theta_{1-i}(\bar{k}),
$$

we may conclude that $A \nvdash \theta_{i}(\bar{k})$, and the proof is complete.

It can be seen that the proofs of Theorems 1 and 2 actually give stronger results than those stated in the theorems. It is also possible to show dual, but necessarily weaker, versions of the theorems. These further results will not, however, be of use in the present paper.

3. Partial Lindenbaum algebras. For the rest of the paper let $n \geq 2$ and let $E=E\left(\Gamma_{n}\right)$ be a partial Lindenbaum algebra for $A$ as defined in the Introduction. The following facts are well known (or trivial).

Facts. (i) $E$ is a countable, dense, distributive lattice with 0 and 1 .

(ii) If $L$ is the full Lindenbaum algebra for an extension $B$ of $P$, then $L \cong E\left(\Delta_{n}\right)$.

(iii) If $A$ is $\Sigma_{1}$-correct, then $E\left(\Sigma_{1}\right), E\left(\Pi_{1}\right)$ and $E$ are pairwise nonisomorphic (in fact not elementary equivalent), since in this case $a \cup b=1$ iff $a=1$ or $b=1$ holds in $E\left(\Sigma_{1}\right)$ and $a \cap b=0$ iff $a=0$ or $b=0$ holds in $E\left(\Pi_{1}\right)$.

Let the reduction principle for $E$ be the following sentence:

$$
\forall a_{0}, a_{1} \exists b_{0}, b_{1}\left(a_{0} \cup a_{1}=b_{0} \cup b_{1} \& b_{0} \cap b_{1}=0 \& b_{0} \leq a_{0} \& b_{1} \leq a_{1}\right),
$$

where 0 is the minimal element of $E$.

THEOREM 3. The reduction principle is false in $E\left(\Pi_{n}\right)$.

PROOF. Let $a_{i}=\left[\neg \sigma_{i}\right]$, where $\sigma_{0}, \sigma_{1}$ are $\Sigma_{n}$-sentences such that $a_{0} \cap a_{1} \neq 0$ (i.e. $\left.A \nvdash \sigma_{0} \vee \sigma_{1}\right)$ and, for $i=0,1, \sigma_{i} \in \operatorname{Cons}\left(\Pi_{n}, A+\neg \sigma_{1-i}\right)$. Such sentences exist by Theorem 1. Suppose now that $\left[\psi_{i}\right]=b_{i} \leq a_{i}$ and that $a_{0} \cup a_{1}=b_{0} \cup b_{1}$. We then have

(i) $A \vdash \psi_{i} \rightarrow \neg \sigma_{i}$,

(ii) $A \vdash \neg \sigma_{0} \vee \neg \sigma_{1} \leftrightarrow \psi_{0} \vee \psi_{1}$.

Thus $A+\neg \sigma_{i}+\sigma_{1-i} \vdash \psi_{i}$ so $A+\neg \sigma_{i} \vdash \psi_{i}$ and thereby $A \vdash \neg \sigma_{i} \leftrightarrow \psi_{i}$. But then $b_{0} \cap b_{1}=a_{0} \cap a_{1} \neq 0$.

It is not difficult to show that the reduction principle is true in $E\left(\Sigma_{n}\right)$, by simply imitating the usual proof of the corresponding recursion theoretic principle (cf. [5]). 
We thus have the main result of this section, namely

COROLlaRY 1. $E\left(\Sigma_{n}\right) \not \equiv E\left(\Pi_{n}\right)$, and thus $E\left(\Sigma_{n}\right) \not \equiv\left(\Pi_{n}\right)$.

Finally we state two open problems:

(A) Is $E\left(\Gamma_{n}\right)$ homogeneous in the sense that all intervals are isomorphic?

(B) Let $E^{A}\left(\Gamma_{n}\right)$ and $E^{B}\left(\Gamma_{m}\right)$ be partial Lindenbaum algebras for two (possibly identical) extensions of $P$. When does $E^{A}\left(\Gamma_{n}\right) \cong E^{B}\left(\Gamma_{m}\right)$ hold?

That the two questions are related may be seen from the fact that an interval $\left[a_{0}, a_{1}\right]$ in $E^{A}\left(\Gamma_{n}\right)$ is isomorphic to $E^{B}\left(\Gamma_{n}\right)$, where $B=A+\neg \varphi_{0}+\varphi_{1}$ for $\left[\varphi_{i}\right]=a_{i}$.

\section{REFERENCES}

1. G. Birkhoff, Lattice theory, 3rd ed., Amer. Math. Soc. Colloq. Publ., vol. 25, Amer. Math. Soc., Providence, R. I., 1967.

2. S. Feferman, Arithmetization of metamathematics in a general setting, Fund. Math. 49 (1960), 33-92.

3. D. Guaspari, Partially conservative extensions of arithmetic, Trans. Amer. Math. Soc. 254 (1979), 47-68.

4. P. Lindström, On partially conservative sentences and interpretability, Proc. Amer. Math. Soc. 91 (1984), 436-443.

5. H. Rogers, Theory of recursive functions and effective computability, McGraw-Hill, New York, 1967.

Department of Philosophy, University of Göteborg, 41298 Göteborg, SWeDEN 